\title{
Diffusion-Weighted Magnetic Resonance Imaging (DW-MRI) as a Diagnostic Method for Prostate Cancer
}

\author{
Mohsen Sarkarian ${ }^{1}$, Alireza Kheradmand ${ }^{1}$, Hamed Haghiabi ${ }^{2,{ }^{*}}$ and Mohammad Kolbadinezhad ${ }^{2}$ \\ ${ }^{1}$ Department of Urology, Golestan Hospital, Ahvaz Jundishapur University of Medical Sciences, Ahvaz, Iran \\ ${ }^{2}$ Ahvaz Jundishapur University of Medical Sciences, Ahvaz, Iran \\ "Corresponding author: Urology Resident, Ahvaz Jundishapur University of Medical Sciences, Ahvaz, Iran. Tel: +98-9184374727, Email: mahmoodrezaie069@gmail.com
}

Received 2018 August 02; Accepted 2018 August 20.

\begin{abstract}
Background: Prostate cancer is the second most common cancer among men in the world. Due to the slow growth of this cancer, its early detection can lead to definitive treatment and mortality reduction. Based on the lower diagnostic ability of conventional methods, imaging methods have been paid attention in recent years.

Objectives: The aim of this study was to evaluate the value and accuracy of diffusion MRI in the prostate cancer as a diagnostic method.

Methods: This was a descriptive cross-sectional study conducted in a 15-month period in patients with prostate cancer who had lower urinary tract symptoms, high PSA (higher than $4 \mathrm{ng} / \mathrm{mL}$ ), or an abnormal finding in the digital rectum examination. The patients were evaluated by MRI of the whole prostate gland with T2 weighted and diffusion images. Patients' conditions were determined by a urologist with standard 12-fold transrectal sonography-guided biopsy of the prostate. Then, by a histologic examination, healthy or cancerous tissues, Gleason score, and the positive blind numbers were determined by a pathologist. In the end, the results were compared.

Results: We observed 80 patients among whom, 25 cases (31.2\%) had malignancies in DW-MRI, including 22 cases that had a malignancy in biopsy and three cases whose biopsy indicated the benign pathology. MRI findings in 55 cases (68.7\%) were benign (BPH, prostatitis) that in four cases, malignancy was seen in the biopsy. The sensitivity of DW-MRI was $84 \%$ and specificity was $94 \%$, indicating a high diagnostic capability for DW-MRI. The positive and negative predictive values of DW-MRI for prostate cancer were $88 \%$ and $92 \%$, respectively. We also observed that older age leads to the lower sensitivity of the MRI. Gleason score, PSA level, and tumor burden had a direct relationship with the accuracy of DW-MRI. However, prostate volume did not have a significant relationship with the detection of cancer in MRI.

Conclusions: DW-MRI can be used for better diagnosis and staging of prostate cancer in patients with prostate lesions. Accordingly, it can upgrade cancer diagnosis in patients with negative biopsies and high suspicion of cancer.
\end{abstract}

Keywords: Magnetic Resonance Diffusion, Diagnosis, Prostate Cancer

\section{Background}

Prostate cancer is the second most common cancer among men in the world. This cancer is also the second leading cause of all deaths from cancer. Due to the slow growth of this cancer, early detection can lead to definitive treatment and mortality reduction. Considering the low diagnostic potential of conventional screening methods for prostate cancer, including prostatic specific antigen (PSA), digital rectal examination (DRE), and transrectal ultrasound-guided biopsy (TRUS-Bx) in the diagnosis and localization of tumors, the use of imaging methods recently has been paid attention (1-3). However, the cancer detection is still not satisfactory. Although PSA has a high sensitivity, its specificity decreases when the PSA level is low (4-10 ng/mL); that is, false positive PSA reduces as the rise in the PSA may occur in some non-cancerous conditions, such as benign hyperplasia and chronic inflammation of the prostate $(4,5)$.

Because tissue sampling is random, it is possible that clinically important lesions outside of the sampling area are not well detected. In addition, a biopsy is commonly made of the peripheral areas of the prostate; thus, lesions in the transient or anterior prostate may not be well detected. Among the methods of MRI, using endorectal coil is known as the best imaging method for the diagnosis and staging of prostate cancer $(6,7)$.

Conventional magnetic resonance imaging (T2weighted MRI) provides excellent anatomical information 
about the prostate. However, despite a high sensitivity in detecting prostate cancer, other lesions including chronic prostate inflammation and benign prostatic hyperplasia appear to be similar to malignant prostate cancer; so, its specificity is low $(8,9)$. Functional MRI techniques can provide a better distinction between benign and malignant tumors. One of these techniques promising for prostate cancer diagnosis is the diffusion weighted MRI (DW-MRI), which measures intracellular and extracellular Browning motions and the distribution of water molecules in biological tissues. By using this imaging technique, we can obtain information about the cellular structure of the tissue that helps differentiate the cancerous tissue from normal and inflammatory tissues $(6,10)$. Accordingly, since other diagnostic methods can diagnose this condition, MR diffusion has been suggested for better evaluation of patients as a low invasive method.

\section{Objectives}

In our study, we applied the MR diffusion as an approach to evaluate patients with prostate lesions to find a better diagnostic approach for prostate cancer.

\section{Methods}

\subsection{Study Setting}

It was a hospital-based study conducted on patients with prostate cancers.

\subsection{Study Population}

The study population included all patients with a prostate lesion diagnosis. Accordingly, in a 15-month period, 80 patients who were suspicious of prostate cancer were considered to form the study group. This case group was made of patients with lower urinary tract symptoms, PSA of more than $4 \mathrm{ng} / \mathrm{mL}$, or abnormality in the digital rectal examination.

\subsection{Measurements}

The patients were evaluated by MRI of seminal vesicle, prostate gland, and bladder in sagittal, coronal, and axial sections by a radiologist. In addition, the patients were evaluated by a transrectal sonography and 12 prostate biopsies were taken and based on the Gleason index, the malignancy status of the prostate was determined. The standard 12-fold transrectal sonography-guided biopsies of the prostate were done by a urologist. In addition, histologic examination, healthy or cancerous tissues, Gleason scores, and the positive blind numbers were determined by a pathologist. Then, the results were compared.

\subsection{Ethical Considerations}

Ethical issues were completely observed by the researchers. The study group adhered to the principles of medical ethics introduced by the Health Ministry, the Declaration of Helsinki, and enactments of the medical ethics committee of Ahvaz University of Medical Sciences. In addition, the ethics committee of Ahvaz University of Medical Sciences approved the protocol of the study. Imaging was not supposed to have an effect on the patient's treatment process and the results of the assessment were only reported to the patient.

\subsection{Statistical Analysis}

The data were analyzed by SPSS program and $\mathrm{P}<0.05$ was considered significant. We used $t$-test for quantitative variables and $\kappa 2$ test for qualitative variables.

\section{Results}

We evaluated 80 patients as the study group in which, 28 cases (35\%) suffered from malignancies and 52 (65\%) cases had benign masses. Of the benign cases, 44 cases (55\%) had BPH and eight (10\%) cases had prostatitis (Table 1). The main aim of our study was to investigate the accuracy of magnetic resonance imaging (diffusion MRI) in the diagnosis of prostate cancer. On this basis, we found that the sensitivity of this test was $84 \%$, specificity was $94 \%$, the positive predictive value was $88 \%$, and the negative predictive value was $92 \%$ in the detection of prostate cancer (Table 2 ).

In addition, we observed that older age was associated with the lower sensitivity of the MRI, the sensitivity of the test in the age range of fewer than 65 years was $90 \%$, in the age range of 65 to 85 years was $82 \%$, and in more than 85 years of age was $78 \%$, which showed a statistically significant difference $(\mathrm{P}<0.05)$. In addition, we evaluated the sensitivity of this diagnostic test after comparing the sensitivity of this test with PSA levels. Based on this, we observed

\begin{tabular}{lc}
\hline Table 1. Diagnosis of Evaluated Patients in the Study & \\
\hline Diagnosis & Patients, No. (\%) \\
\hline Malignancy & $28(35)$ \\
BPH & $44(55)$ \\
Prostatitis & $8(10)$ \\
\hline & \\
\hline Table 2. The Accuracy of DW MRI in the Diagnosis of Prostate Cancer \\
\hline Variables & Percentage \\
\hline Sensitivity & 84 \\
\hline Specificity & 94 \\
\hline Positive predictive value & 88 \\
\hline Negative predictive value & 92 \\
\hline
\end{tabular}




\begin{tabular}{|c|c|c|}
\hline Variables & Sensitivity (\%) & PValue \\
\hline Age $(y)$ & & $<0.05$ \\
\hline Lower than 65 & 90 & \\
\hline $66-85$ & 82 & \\
\hline More than 86 & 78 & \\
\hline PSA & & $<0.05$ \\
\hline 4-10 or Lower & 80 & \\
\hline More than 10.1 & 92 & \\
\hline Gleason score & & $<0.05$ \\
\hline 6 or lower & 79 & \\
\hline 7 & 85 & \\
\hline 8 or higher & 100 & \\
\hline Number of biopsies & & $<0.05$ \\
\hline 1 & 80 & \\
\hline 2 & 84 & \\
\hline 3 or more & 91 & \\
\hline
\end{tabular}

that if this antigen was at the level of 4 to 10 , the sensitivity would be less than $80 \%$ while a PSA level of greater than 10 was associated with the sensitivity of greater than $92 \%$, and this showed a statistically significant difference in patients $(\mathrm{P}<0.05)$. In addition, we observed that the Gleason score (GS) had a direct relationship with the sensitivity of this test as increases in the GS could increase the sensitivity of this test. Accordingly, if the GS was less than six, the sensitivity of the test would be $79 \%$, in the GS of seven, the sensitivity would be $85 \%$, and in the GS of more than or equal to eight, the sensitivity would be $100 \%$; this showed a statistically significant difference in patients $(\mathrm{P}<0.05)$. In addition, based on the number of biopsies, we found that in one biopsy, the sensitivity was $80 \%$, in two biopsies, it was $84 \%$, and in more than two biopsies, it was $91 \%$, and this showed a significant difference between the sensitivity values of DWMRI $(\mathrm{P}<0.05)$ (Table 3$)$.

\section{Discussion}

We found that the diffusion MRI could be used where multi-parametric MRI is not possible to perform. In the following, other studies conducted on this diagnostic method are discussed. In a study, Wu et al. found that the sensitivity and specificity of DWI images associated with T2 weight in detecting the prostate cancer were $72 \%$ and $81 \%$, and the sensitivity and specificity of T2-weighted images alone in detecting the prostate cancer were $62 \%$ and $77 \%$, respectively. This result is consistent with the results of our study and shows the effectiveness of this test in assessing patients (10). Tan et al. also stated in their assessment that the sensitivity and specificity of DWI images alone (69\% and $89 \%$, respectively) and DWI + T2-weighted images (70\% and $83 \%$, respectively) were higher than the sensitivity and specificity of T2-weighted images alone (60\% and 76\%, respectively), which is consistent with the observed results in our assessment (8). Aydin et al. compared the accuracy of five types of MRI (diffusion, T1, T2, contrast dynamics, and spectroscopy) with transrectal biopsy and concluded that using a combination of these sequences instead of using any of these methods alone increases the diagnostic accuracy and its efficacy in the staging of the prostate cancer (1). Haider et al. also stated in a study that the accuracy of DWI + T2-weighted images was significantly more than that of T2-weighted images (81\% vs. 54\%) and generally stated that the DWI + T2-weighted images in detecting advanced cancer (a Gleason score of above 6 and a diameter of more than $4 \mathrm{~mm}$ ) in the peripheral area is more effective than T2-weighted images (11).

Hricak et al. in their assessment of various modalities of prostate cancer stated that the accuracy of MRI in the staging of prostate cancer is between 54\% and 93\% (12). Chen et al. compared the accuracy of three types of MRI (diffusion, T2, and spectroscopy) with transrectal biopsy in the diagnosis of prostate cancer and observed that the accuracy of prostate cancer detection increased with a combination of all three methods (13). Shimofusa et al. in a study in 2005 stated that the accuracy of MRI for diagnosing prostate cancer was $87 \%$ and $93 \%$ with DWI. According to the study, using DWI in MRI images increased the accuracy of the cancer diagnosis (14). Therefore, most studies in this area are consistent with the results of our assessment, which indicates the effectiveness of this test in evaluating patients with prostate lesions.

\subsection{Conclusions}

Since diffusion MRI is a non-aggressive test, multiparametric MRI is not available at most centers and cities, and the cost of this imaging is too high, the diffusion MRI due to its acceptable accuracy can be used where multiparametric MRI is not possible to perform. Thus, this diagnostic test can be used to better understand the patients' conditions and accordingly, to upgrade the diagnostic methods to be used for patients with prostate lesions.

\section{References}

1. Aydin H, Kizilgoz V, Tatar IG, Damar C, Ugan AR, Paker I, et al. Detection of prostate cancer with magnetic resonance imaging: Optimization of T1-weighted, T2-weighted, dynamic-enhanced T1weighted, diffusion-weighted imaging apparent diffusion coefficient mapping sequences and MR spectroscopy, correlated with biopsy and histopathological findings. J Comput Assist Tomogr. 2012;36(1):30-45. doi: 10.1097/RCT.0b013e31823f6263. [PubMed: 22261768]. 
2. Chan JM, Stampfer MJ, Giovannucci E, Gann PH, Ma J, Wilkinson P, et al. Plasma insulin-like growth factor-I and prostate cancer risk: A prospective study. Science. 1998;279(5350):563-6. [PubMed: 9438850].

3. Moul JW, Sesterhenn IA, Connelly RR, Douglas T, Srivastava S, Mostofi FK, et al. Prostate-specific antigen values at the time of prostate cancer diagnosis in African-American men. JAMA. 1995;274(16):1277-81. [PubMed: 7563532].

4. Heidenreich A, Bastian PJ, Bellmunt J, Bolla M, Joniau S, van der Kwast T, et al. EAU guidelines on prostate cancer. part 1: Screening, diagnosis, and local treatment with curative intent-update 2013. EurUrol.2014;65(1):124-37. doi:10.1016/j.eururo.2013.09.046.[PubMed: 24207135].

5. Welch HG, Albertsen PC. Prostate cancer diagnosis and treatment after the introduction of prostate-specific antigen screening: 1986 2005. J Natl Cancer Inst. 2009;101(19):1325-9. doi: 10.1093/jnci/djp278. [PubMed: 19720969]. [PubMed Central: PMC2758309].

6. Sweeney CJ, Chen YH, Carducci M, Liu G, Jarrard DF, Eisenberger $\mathrm{M}$, et al. Chemohormonal therapy in metastatic hormone-sensitive prostate cancer. $N$ Engl J Med. 2015;373(8):737-46. doi: 10.1056/NEJMoa1503747. [PubMed: 26244877]. [PubMed Central: PMC4562797].

7. Afshar-Oromieh A, Avtzi E, Giesel FL, Holland-Letz T, Linhart HG, Eder $\mathrm{M}$, et al. The diagnostic value of PET/CT imaging with the (68)Ga-labelled PSMA ligand HBED-CC in the diagnosis of recurrent prostate cancer. Eur J Nucl Med Mol Imaging. 2015;42(2):197-209. doi:10.1007/s00259-014-2949-6. [PubMed: 25411132]. [PubMed Central: PMC4315487].

8. Tan CH, Wei W, Johnson V, Kundra V. Diffusion-weighted MRI in the detection of prostate cancer: Meta-analysis. AJR Am J Roentgenol.
2012;199(4):822-9. doi: 10.2214/AJR.11.7805. [PubMed: 22997374] [PubMed Central: PMC3888871].

9. Kenfield SA, Stampfer MJ, Giovannucci E, Chan JM. Physical activity and survival after prostate cancer diagnosis in the health professionals follow-up study. J Clin Oncol. 2011;29(6):726-32. doi: 10.1200/JCO.2010.31.5226. [PubMed: 21205749]. [PubMed Central: PMC3056656].

10. Wu LM, Xu JR, Ye YQ, Lu Q, Hu JN. The clinical value of diffusionweighted imaging in combination with T2-weighted imaging in diagnosing prostate carcinoma: A systematic review and meta-analysis. AJR Am J Roentgenol. 2012;199(1):103-10. doi: 10.2214/AJR.11.7634. [PubMed: 22733900].

11. Haider MA, van der Kwast TH, Tanguay J, Evans AJ, Hashmi AT, Lockwood G, et al. Combined T2-weighted and diffusion-weighted MRI for localization of prostate cancer. AJR Am J Roentgenol. 2007;189(2):323-8. doi: 10.2214/AJR.07.2211. [PubMed: 17646457].

12. Hricak H, Choyke PL, Eberhardt SC, Leibel SA, Scardino PT. Imaging prostate cancer: A multidisciplinary perspective. Radiology. 2007;243(1):28-53. doi: 10.1148/radiol.2431030580. [PubMed: 17392247].

13. Chen M, Dang HD, Wang JY, Zhou C, Li SY, Wang WC, et al. Prostate cancer detection: Comparison of T2-weighted imaging, diffusionweighted imaging, proton magnetic resonance spectroscopic imag ing, and the three techniques combined.Acta Radiol.2008;49(5):60210. doi: 10.1080/02841850802004983. [PubMed: 18568549].

14. Shimofusa R, Fujimoto H, Akamata H, Motoori K, Yamamoto S, Ueda T, et al. Diffusion-weighted imaging of prostate cancer. J Comput Assist Tomogr. 2005;29(2):149-53. [PubMed: 15772529]. 